\title{
Aerodynamic Sound Radiated from Two-Dimensional Airfoil with Local Porous Material
}

\author{
Hiromitsu Hamakawa ${ }^{1}$, Kazuki Hosokai ${ }^{2}$, Takaaki Adachi ${ }^{2}$, Eru Kurihara ${ }^{1}$ \\ ${ }^{1}$ Oita University, Oita, Japan \\ ${ }^{2}$ Postgraduate Course, Oita University, Oita, Japan \\ Email: hamakawa@oita-u.ac.jp
}

Received May 28, 2013; revised June 5, 2013; accepted June 12, 2013

Copyright (C) 2013 Hiromitsu Hamakawa et al. This is an open access article distributed under the Creative Commons Attribution License, which permits unrestricted use, distribution, and reproduction in any medium, provided the original work is properly cited.

\begin{abstract}
In the present paper, the attention is focused on the effect of local porous material on aerodynamic sound radiated from two-dimensional airfoil. We measured the aerodynamic sound radiated from the airfoil with porous material, tripping wire and porous plate which are mounted locally on the surface of the airfoils near the leading edge. At the normal airfoil, discrete frequency noise is clearly observed at small attack angle. However, it is clear that its noise generated from the airfoil decreased with the local porous material on the surface of pressure side of the airfoil. The porous material is effective to reduce this noise compared with the others. And the sound absorbing coefficient and the air permeability were measured for test porous material. The sound absorbing coefficient increased at the high frequency band, and the air permeability became small for porous materials. As the attack angle increased, the discrete frequency noise was not generated from the normal airfoil. The broadband noises were almost same for all test airfoils.
\end{abstract}

Keywords: Aerodynamic Sound; Discrete Frequency Noise; Porous Material; Two-Dimensional Airfoil

\section{Introduction}

Low noise level is an important sales point of the various kinds of machines as well as high performance and miniaturization. This situation is also applied to fans used, for example, in air conditioners, ventilators and coolers.

The controlling noise source generated from an axial flow fan is turbulent noise due to vortex shedding when the fan is operated near the design point $[1,2]$. Fukano et $a l$. have investigated the discrete frequency noise generated by Karman vortex shedding from a flat plate blade immersed in a uniform two-dimensional flow field, and theoretically introduced a formula to predict its sound pressure level [3].

It is generally known that the tonal noise is generated from two-dimensional airfoil at certain flow conditions at a discrete frequency about $30 \mathrm{~dB}$ above the background broadband level. This discrete frequency noise is commonly generated from fans, wind-turbines, gliders and small aircrafts, etc. Many studies have been published on the characteristics and occurrence mechanisms of this noise [4-10].

On the other hand, there are many studies to reduce the aerodynamic noise radiated from the airfoil [11-15]. Fukano et al. also reported that the fan noise decreased by changing the profile of rotor blade [11]. Polacsek et al. showed that the wavy-leading-edge of blade was effecttive to reduce its noise [12]. Nishimura et al. observed that the fan noise reduced to affix a fur material around leading edge on the surface of blade for cooling fan [13]. However, the aerodynamic noise may increase when the rotational speed of fan increases to supplement the decrease of fan performance, although an aerodynamic noise decreases for these methods. Therefore, it is considered that the reduction of aerodynamic noise by changing the properties on the surface of the blade, which is not changing the blade profile, is effective, because the design of high efficiency is more important for industrial fans. Akishita et al. [14] and Takeishi et al. [15] have clarified the acoustic characteristic on the porous surface and the effect of porous surface on Aeolian tone radiated from a circular cylinder. However, the specifications of the porous material on the blade, for example, properties, hole diameter, thickness and optimum position, etc. are not clear, although it may be effective to reduce an aerodynamic sound without decreasing the performance of fan.

The purpose of the present study is to clarify the effect of the local porous material mounted near the leading 
edge on the surface of airfoil on an aerodynamic sound. And the characteristics of the air permeability and sound absorbing coefficient of porous materials are also discussed.

\section{Experimental Apparatus and Procedures}

\subsection{Measurement of Aerodynamic Sound}

Our experiments were performed in a low-noise wind tunnel, which has been described in detail elsewhere [16]. This wind tunnel was an open-circuit with wing-type silencers in the diffuser located at the outlet of the blower and splitter-type silencers at the inlet of the blower. The test section was placed in the anechoic room, which was rectangular in shape, and $3 \mathrm{~m}$ long, $3 \mathrm{~m}$ wide, and $3 \mathrm{~m}$ high. The collector was downstream of the test section. Noise-absorbing furry materials were attached to the surface of the collector to reduce the interaction noise between the open jet and the collector. This collector was connected to a 3-m-long sound absorbent duct. The background noise was about $63 \mathrm{~dB}(\mathrm{~A})$ at a freestream velocity of $50.0 \mathrm{~m} / \mathrm{s}$.

Figure 1 shows a schematic view of the test-section and test airfoil. The cross section of the nozzle exit was a 0.3-m-wide and 0.3-m-high square. A test airfoil was installed in the test section $100 \mathrm{~mm}$ downstream of the nozzle exit. The freestream velocity ranged from $5 \mathrm{~m} / \mathrm{s}$ to $45 \mathrm{~m} / \mathrm{s}$ at the test section inlet. The Reynolds numbers, based on the chord length, $\ell$, and freestream velocity, $U_{\infty}$, ranged from $3.1 \times 10^{4}$ to $3.4 \times 10^{5}$. The flow past the nozzle was uniform, and the drift of the freestream velocity was less than about $0.9 \%$. The freestream turbulence level was less than $0.5 \%$ of the freestream velocity. In addition, no peak of velocity fluctuation spectrum at the test section without the test airfoil was formed at this velocity range. Two end plates were placed at the top and bottom of the test section, and a test airfoil was placed vertically and rigidly supported between them. These were 900-mm-wide and 450-mm-long acoustically nonreflecting end plates, which were large enough to cover the jet edge region. The downstream distance from the test airfoil to the edges of the end plates was $350 \mathrm{~mm}$.

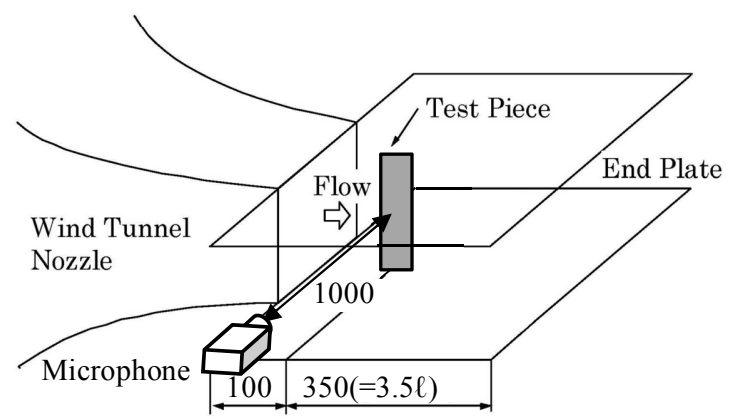

Figure 1. Schematic of test section of wind tunnel.
These end plates were composed of a 25-mm-thick polystyrene porous material and 25-mm-thick glass wool backed with a punched steel plate to reinforce the plate rigidity [16]. It was clearly observed that the results for the non-reflecting end plates were almost the same as the attenuation characteristics of the free field.

Figure 2 shows the test airfoils. The porous plate was mounted near the leading edge on the surface of test airfoil as shown in Figure 2(a) and was made from a 0.2 mm-thick plate with many holes of diameter, $d_{0}$, of about $0.5 \mathrm{~mm}$. The thickness of the background air space is about $4 \mathrm{~mm}$. The airfoil has NACA0012 profile, the chord length is $100 \mathrm{~mm}$ and span length is $300 \mathrm{~mm}$.

The Styrofoam or Polyethylene foam as porous material was mounted near the leading edge on the surface of test airfoil as shown in Figure 2(b) and was thickness of about $4 \mathrm{~mm}$. The tripping wire was mounted near the leading edge on the surface of test airfoil as shown in Figure 2(c) and was diameter, $d$, of $2.6 \mathrm{~mm}$ or $0.8 \mathrm{~mm}$. The Polyethylene foam was mounted on the both surfaces of test airfoil as shown in Figure 2(d). The normal airfoil of NACA0012 profile without porous materials was shown in Figure 2(e). The features and symbols of test airfoils are presented in Table $\mathbf{1}$.

The aerodynamic sound in the far field from the test airfoil was measured at $X=0 \mathrm{~mm}, Y=1000 \mathrm{~mm}$, and $Z=$

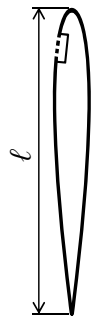

(a)

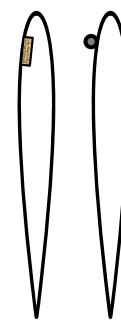

(b)

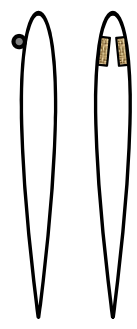

(c)

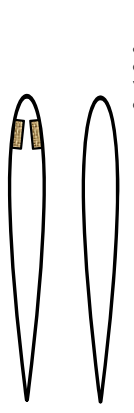

(d) (e)

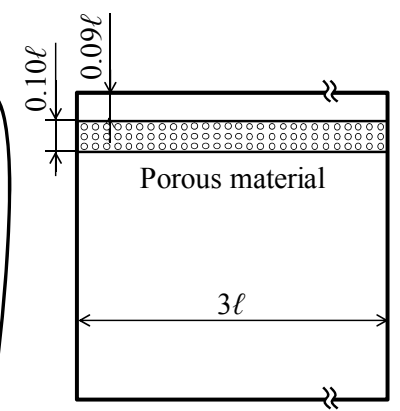

(a)
Figure 2. Test airfoils.

Table 1. Experimental materials.

\begin{tabular}{lll}
\hline Symbols & Feature & Figure \\
\hline Plate & Porous plate, $d_{0}=0.5 \mathrm{~mm}$ & Figure 2(a) \\
Material A & Porous material, Styrofoam & Figure 2(b) \\
Material B & Porous material, Polyethylene foam & Figure 2(b) \\
Trip 2.6 & Tripping wire, $d=2.6 \mathrm{~mm}$ & Figure 2(c) \\
Trip 0.8 & Tripping wire, $d=0.8 \mathrm{~mm}$ & Figure 2(c) \\
Both Sides & Material B on the both sides & Figure 2(d) \\
Normal & Normal type & Figure 2(e) \\
\hline
\end{tabular}


$0 \mathrm{~mm}$ using a microphone. When the observation location was far enough to be considered as the far field, the effect of the near field could be neglected. In this measuring position, the near field component attenuates, and the far field component is about $10 \mathrm{~dB}$ larger than the near field component for phenomena that occur over 170 $\mathrm{Hz}$.

The microphone output was sampled by an FFT analyzer and the statistical parameters were calculated. The spectra of the sound pressure level (SPL) were calculated for 80 ensemble averages of 2048 data points that were sampled at $12.8 \mathrm{kHz}$. The frequency resolution was estimated to be $12.5 \mathrm{~Hz}$.

We measured SPL at attack angle, $\alpha$, from -30 to 30 degree of $R_{e}=1.5 \times 10^{5}$. The position of porous material on the airfoil is shown in Figure 3. When the porous material mounts on the surface of suction side of test airfoil, $\alpha$ is positive value. Therefore, when $\alpha$ is negative value, the porous material is located on the surface of pressure side of airfoil.

\subsection{Measurement of Air Permeability}

The air permeability rate is in proportion to the pressure drop, time, and area of porous material, and is in inverse proportion to the thickness of porous material. Therefore, the air permeability was defined by

$$
\mu=\frac{Q}{\Delta P} \frac{\delta}{A}
$$

where $Q$ is the volume flow rate, $\delta$ is the thickness of porous material, $\Delta P$ is the pressure drop, $A$ is the area of porous material. The experimental apparatus of air permeability measurement was shown in Figure 4. $\mu$ were calculated from these values measured by the sensors of test apparatus in Figure 4. The measured air permeability, $\mu$, for test porous materials are shown in Figure 5. $\mu$ of Styrofoam (Material A) as porous material became about 10 times larger than that of porous plate (Plate). And $\mu$ of porous plate (Plate) was similar with the results of Polyethylene foam (Material B) at the low flow rate, $Q$.

\subsection{Measurement of Sound Absorbing Coefficient}

The experimental apparatus of acoustic impedance measurement was shown in Figure 6. A test porous material was enclosed within the test apparatus into which an acoustic wave was emitted from the loudspeaker. The

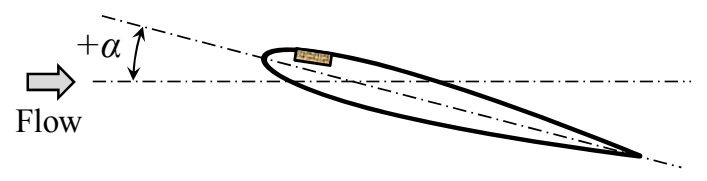

Figure 3. Attack angle and position of porous material.

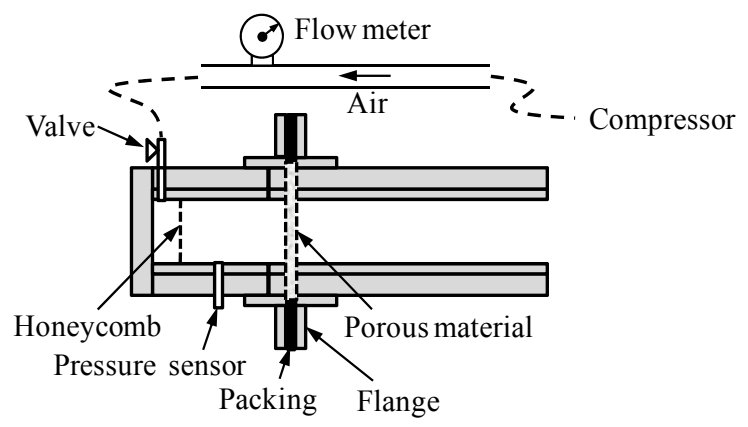

Figure 4. Schematic of test apparatus of air permeability.

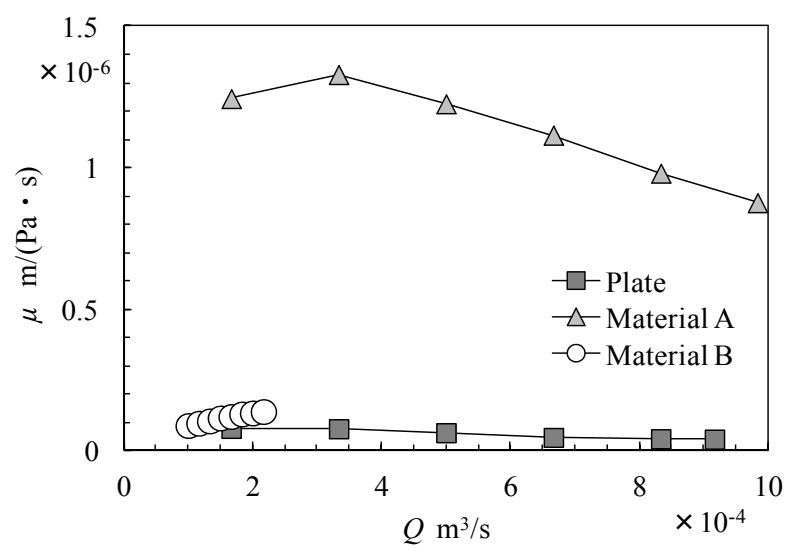

Figure 5. Air permeability against volume flow rate.

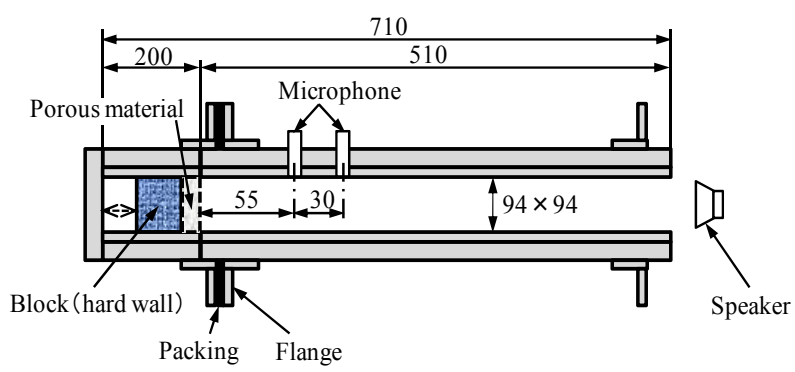

Figure 6. Schematic of acoustic impedance tube.

transfer function was measured according to ISO10534-2. The normal incidence absorption coefficient and acoustic characteristics were calculated from the obtained transfer function by using two microphone methods. Its measureing range is $100-1500 \mathrm{~Hz}$ for a large tube of an internal cross section of $94 \times 94 \mathrm{~mm}$, and $1500-5000 \mathrm{~Hz}$ for a small tube of $24 \times 24 \mathrm{~mm}$.

Figure 7 shows the absorption coefficients, $\beta$, for test porous materials. The gray dotted line represents the result for the porous plate. $\beta$ became high value of 1.0 between about $3000 \mathrm{~Hz}$ to $4000 \mathrm{~Hz}$. The dark solid line and dotted line are results of Polyethylene foam and Styrofoam respectively. $\beta$ of Styrofoam agreed well with that of Polyethylene foam. $\beta$ of these materials became about 1.0 about $4800 \mathrm{~Hz}$. As frequency, $f$, decreased, $\beta$ of these materials decreased. 


\section{Results and Discussion}

\subsection{Discrete Frequency Noise Radiated from Normal Airfoil}

First, we measured the aerodynamic sound radiated from normal airfoil without porous material to clarify the effect of porous material on its sound. The gray solid line in Figure 8 shows a typical spectrum of sound pressure level (SPL) at attack angle, $\alpha$, of 0 degree. Multiple peaks observed in the spectrum of SPL.

Figure 9 shows variation of these peak frequencies of SPL against freestream velocity, $U_{\infty}$. The peak frequencies were dependent on the freestream velocity $U_{\infty}^{1.5}$. For small variations in the freestream velocity, the frequencies

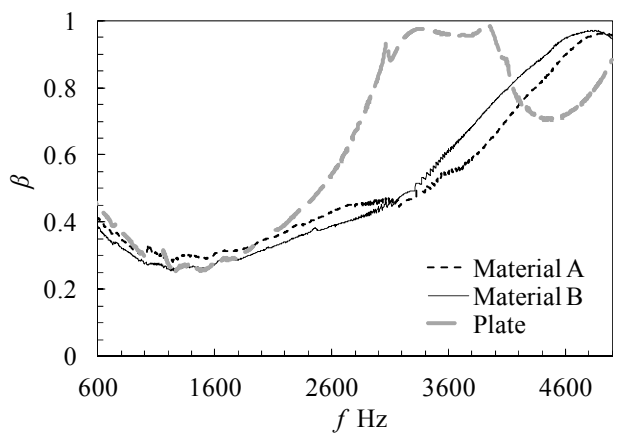

Figure 7. Sound absorbing coefficient.

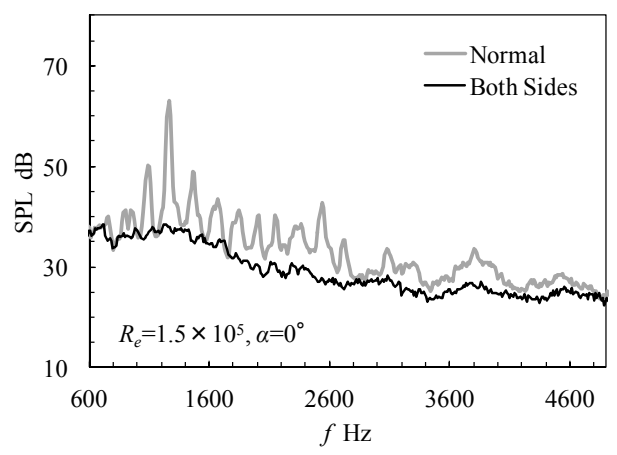

Figure 8. Spectra of SPL of test blades.

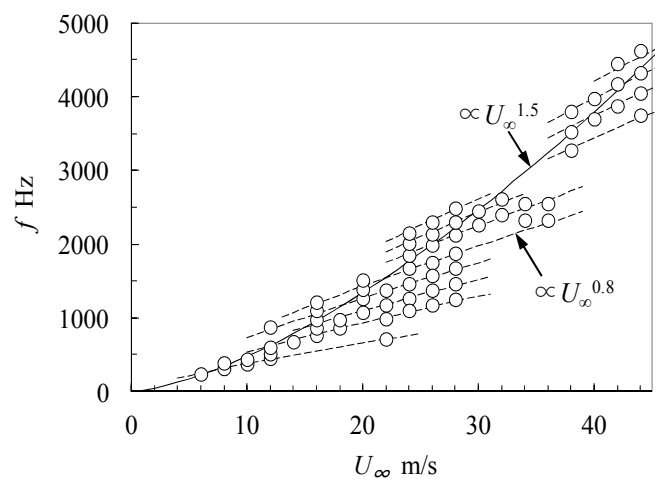

Figure 9. Variation of peak frequencies against freestream velocity. of these sounds were approximately proportional to $U_{\infty}^{0.8}$. At intermittently spaced freestream velocities the frequency of the sound was observed to jump to other curves proportional to $U_{\infty}^{1.5}$. These are same tendency for Paterson et al. [4].

\subsection{Effect of Local Porous Material on Aerodynamic Sound}

The spectra of SPL of the aerodynamic sound radiated from the test airfoils at $\alpha=-2$ degree were measured. The porous materials are mounted at the surface of the pressure side of the test airfoils. The dotted line in Figure 10 shows the baseline spectrum of SPL for normal airfoil. Multiple peaks observed in the spectrum as well as the results of $\alpha=0$ degree. The red line and blue line in Figure 10 show the spectra of SPL for Material B and Plate respectively. No peaks were formed in these spectra. The maximum peak level is approximately $51.7 \mathrm{~dB}$ lower than the normal airfoil at $1237.3 \mathrm{~Hz}$. It is clear that these materials are effective to reduce the discrete frequency noise radiated from airfoil. However, SPL over $2600 \mathrm{~Hz}$ for Plate increased rather than that for Material B. In Figure 10, the green dotted line, purple line and yellow line represent the spectra of SPLs for Material A, Trip 2.6 and Trip 0.8 respectively. The maximum peak level of Material A is approximately $32.4 \mathrm{~dB}$ lower than the normal airfoil although the multiple peaks observed in the spectrum. The tripping wire changes the roughness of the surface of airfoil, and increases the velocity disturbance in the boundary layer. This means that the reduction of peak SPL depends on the intensity of velocity disturbance in the boundary layer on the surface of the pressure side of airfoil. On the other hand, the SPLs are almost same from $2600 \mathrm{~Hz}$ to $4600 \mathrm{~Hz}$ for all test airfoils. It is considered that the peak SPL does not depend on the sound absorption coefficients. The reason for this is that the area of the porous material is small on the surface of airfoil.

Figure 11 shows the spectra of SPL at $\alpha=+2$ degree. The porous materials are mounted at the surface of the

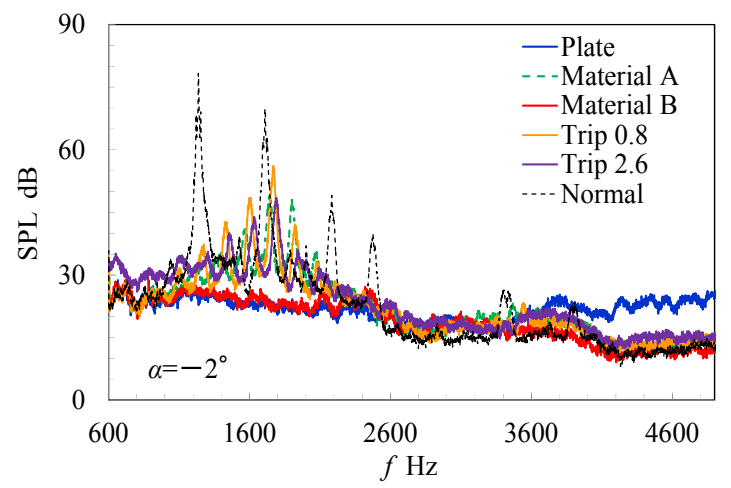

Figure 10. Comparison of spectra of SPL at $\alpha=-2$ degree. 


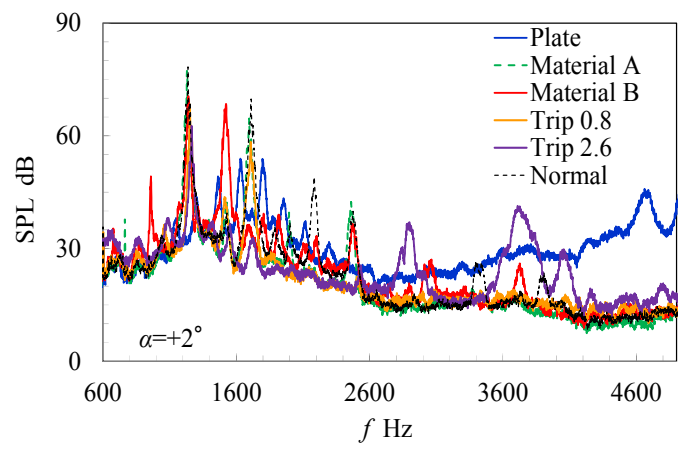

Figure 11. Comparison of spectra of SPL at $\alpha=+2$ degree.

suction side of the test airfoils. The discrete frequency noise was generated for all test airfoils. In specially, SPL over $2600 \mathrm{~Hz}$ for Plate and Trip 2.6 increased rather than that for normal airfoil.

As absolute value of $\alpha$ increased, the discrete frequency noise was not generated for normal airfoil. Figure 12 represents the typical spectra of SPL at $\alpha=-6$ degree for all test airfoils. The discrete frequency noise was not generated. The spectra of SPL were almost same for all test airfoils. It is clear that the porous materials are not effective to reduce the broad-band noise radiated from airfoil at larger $\alpha$. This indicates that the SPL does not also depend on the sound absorption coefficients in this flow condition.

Figure 13 is the results of $\alpha=+6$ degree. The discrete frequency noise was generated for Plate although its noise was not for the others. The peak SPL of discrete frequency noise for Plate increased from $\alpha=+1$ to 9 degree. The Plate was not effective to reduce the discrete frequency noise radiated from airfoil at larger $\alpha$. It is considered that this is caused by the suitable velocity disturbance in the boundary layer generated from the porous plate near the leading edge. As absolute value of $\alpha$ increased more, the discrete frequency noise was not generated for all test airfoils.

From above discussion, it is considered that Material B is the most effective to reduce the discrete frequency noise. Thus, we measured the peak SPL in the case of Both Sides at freestream velocity from $5 \mathrm{~m} / \mathrm{s}$ to $45 \mathrm{~m} / \mathrm{s}$. The Material B was mounted on the both surface of test airfoil as shown in Figure 2(d). The dark solid line in Figure 8 is the typical result of spectrum of SPL. No peaks were formed in the spectrum. The gray squares in Figure 14 are SPL of Both Sides at the peak frequencies for normal airfoil at $\alpha=0$ degree. The peak SPL decreased for all velocity range. The maximum drop of SPL was about $30.7 \mathrm{~dB}$.

\section{Conclusions}

The effect of porous material on aerodynamic sound radiated from airfoil was experimentally investigated. As a

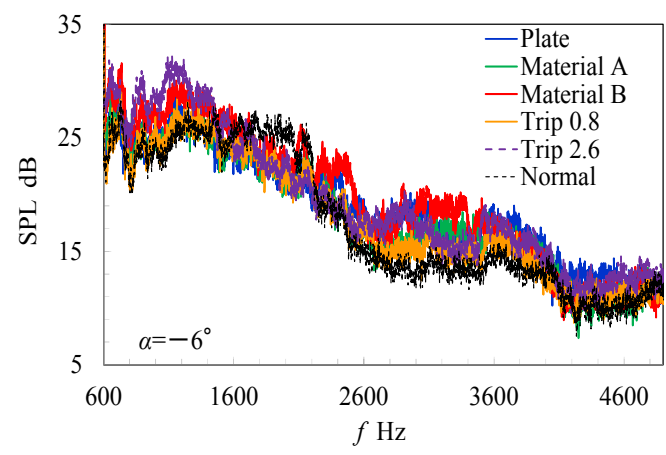

Figure 12. Comparison of spectra of SPL at $\alpha=-6$ degree.

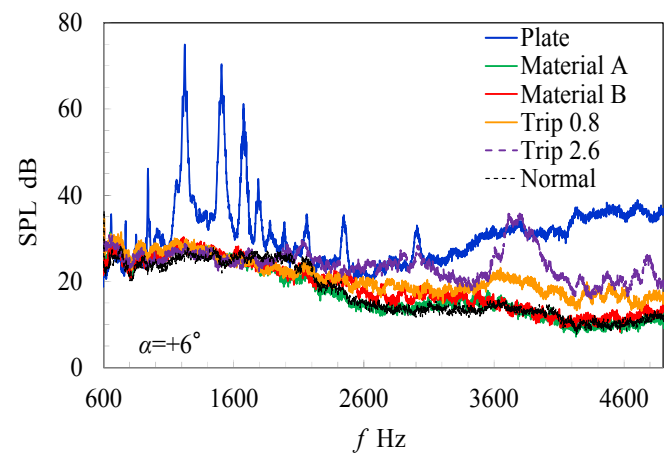

Figure 13. Comparison of spectra of SPL at $\alpha=+6$ degree.

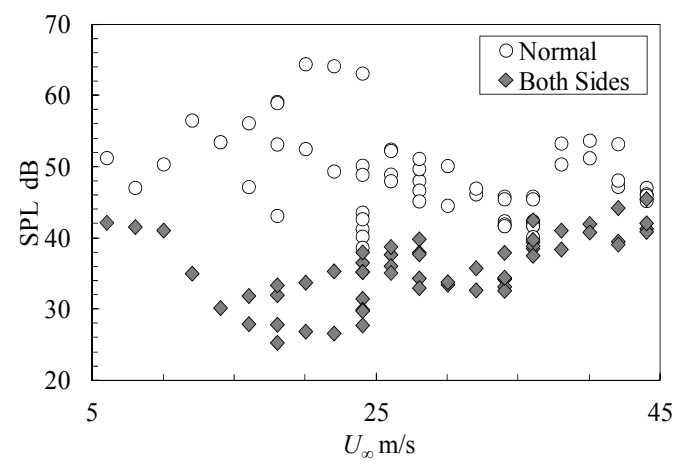

Figure 14. Variation of peak SPL against freestream velocity.

result, the following conclusions were obtained:

1) The Polyethylene foam and the Porous plate are effective to reduce the discrete frequency noise radiated from airfoil at small attack angle. These materials are mounted at the surface of the pressure side near the leading edge of the airfoil. The SPLs of these materials at $1237.3 \mathrm{~Hz}$ are approximately $51.7 \mathrm{~dB}$ lower than that of normal airfoil at attack angle of -2 degree. However, SPL of Porous plate increased rather than that of Poly ethylene foam over $2600 \mathrm{~Hz}$.

2) As absolute value of attack angle increased, the discrete frequency noise was not generated for all test airfoils. The porous materials were not effective to reduce the broad-band noise radiated from airfoil at larger attack 
angle. SPL did not depend on the sound absorption coefficient of porous material.

3) Air permeability of Porous plate was similar with the results of Polyethylene foam at low flow rate. Air permeability of Styrofoam became about 10 times larger than those of Porous plate and Polyethylene foam.

4) Absorption coefficient of Styrofoam agreed well with that of Polyethylene foam. These coefficients for Styrofoam and Polyethylene foam became about 1.0 about $4800 \mathrm{~Hz}$. And as frequency decreased, the absorption coefficients of these materials decreased. This coefficient of Porous plate became high value of 1.0 between about $3000 \mathrm{~Hz}$ to $4000 \mathrm{~Hz}$.

\section{Acknowledgements}

This investigation was supported by a Grant-in-Aid for Scientific Research through grant number 23560265 from Japanese Society for the Promotion of Science.

\section{REFERENCES}

[1] T. Fukano, A. A. Talukuder, T. Kozu and Y. Takamatsu, "Discrete Frequency Noise Generated from a Flat Plate in Parallel with a Uniform Oncoming Flow," Memoirs of the Faculty of Engineering, Kyushu University, Fukuoka, 1984, pp. 19-39.

[2] R. E. Longhouse, "Control of Tip-Vortex Noise of Axial Flow Fans by Rotating Shrouds," Journal of Sound and Vibration, Vol. 58, No. 2, 1978, pp. 201-214.

[3] T. Fukano, Y. Takamatsu and Y. Kodama, "The Effects of Tip Clearance on the Noise of Low Pressure Axial and Mixed Flow Fans," Journal of Sound and Vibration, Vol. 105, No. 2, 1986, pp. 291-308.

[4] R. W. Paterson, P. Vogt, M. Fink and C. Munch, "Vortex Noise of Isolated Airfoils," Journal of Aircraft, Vol. 10, No. 5, 1973, pp. 296-302. doi:10.2514/3.60229

[5] C. K. W. Tam, "Discrete Tones of Isolated Airfoils," Journal of the Acoustical Society of America, Vol. 55, No. 6, 1974, pp. 1173-1177. doi:10.1121/1.1914682

[6] R. E. Longhouse, "Vortex Shedding Noise of Low Tip
Speed, Axial Flow Fans," Journal of Sound and Vibration, Vol. 53, No. 1, 1977, pp. 25-46.

[7] M. R. Fink, "Prediction of Airfoil Tone Frequencies," Journal of Aircraft, Vol. 12, No. 2, 1975, pp. 118-120. doi: $10.2514 / 3.44421$

[8] H. Arbey and J. Bataille, "Noise Generated by Airfoil Profiles Placed in a Uniform Laminar Flow," Journal of Fluid Mechanics, Vol. 134, 1983, pp. 33-47. doi:10.1017/S0022112083003201

[9] S. Nakashima and S. Akishita, "Discrete Tone Noise on Two-Dimensional Wing (Numerical Analysis on Boundary Layer Instability on Wing)," Transactions of JSME, Vol. 66, No. 648B, 2000, pp. 2056-2064.

[10] S. Takagi and Y. Konishi, "Frequency Selection Mechanism of Airfoil Trailing-Edge Noise," Journal of Aircraft, Vol. 47, No. 4, 2010, pp. 1111-1116.

[11] T. Fukano, Y. Kodama and Y. Takamatsu, "Prediction of Sound Pressure Level radiated from Low Pressure Axial Flow Fan and Mixed Flow Fan," Transactions of JSME, Vol. 51, No. 466, 1985, pp. 1825-1832.

[12] C. Polacsek, G. Reboul, V. Clair, T. Le Garrec and H. Deniau, "Turbulence-Airfoil Interaction Noise Reduction using Wavy Leading Edge: An Experimental and Numerical Study," Proceedings of Inter-Noise 2011, Osaka, 4-7 September 2011, 11 pp.

[13] M. Nishimura, T. Goto and T. Ito, "Study on Reducing Noise from a Small Axial Cooling Fan by Using PileFabrics," Proceedings of Internoise 2006, Honolulu, 3-6 December 2006, 9 pp.

[14] S. Akishita, "Grazing Effect of Surface Impedance for Perforated Surface with Very Small Diameter," Proceedings of Inter-Noise 2007, Istanbul, 28-31 August 2007, pp. 1762-1769.

[15] T. Sueki, T. Takaishi and M. Ikeda, "Application of Porous Material to Reduce Aerodynamic Noise Caused by a Pantograph," JSME Annual Meeting 2007, No. 7-1, 2007, pp.71-72.

[16] H. Hamakawa, K. Nakashima and H. Matsue, "Reduction of Noise Generated from Small Wind Tunnel," Proceedings of the Second Asian Joint Workshop on Thermophysics and Fluid Science, Luoyang, 12-14 May 2008, pp. 122-126.

$R_{e}$ : Reynolds number

SPL: Sound pressure level (dB)

$U_{\infty}$ : Freestream velocity $(\mathrm{m} / \mathrm{s})$

$\alpha$ : Attack angle (degree)

$\beta$ : Absorption coefficient

$\delta$ : Thickness of porous material (m)

$\mu$ : Air permeability $(\mathrm{m} /(\mathrm{Pa} \cdot \mathrm{s}))$

$\triangle P$ : Pressure drop $(\mathrm{Pa})$

$Q:$ Volume flow rate $\left(\mathrm{m}^{3} / \mathrm{s}\right)$ 